\title{
DEFINING PEDESTRIAN LEVEL OF SERVICE AT SIGNALIZED INTERSECTIONS THROUGH MODELLED PARAMETERS AND SOM CLUSTERING
}

\author{
Rima Sahani ${ }^{1}$, Vasundhara Devi' ${ }^{2}$ Prasanta Kumar Bhuyan ${ }^{3}$ \\ ${ }^{1}$ Department of Civil Engineering, NIST, Berhumpur, India \\ ${ }^{2,3}$ Deptartment of Civil Engineering, NIT Rourkela, India
}

Received 6 January 2017; accepted 29 September 2017

\begin{abstract}
Aim of this study is to define pedestrian levels of service (PLOS) categories (A-F) at signalized intersections under the influence of mixed traffic flow. Both quantitative and qualitative factors affecting the service measure have been identified and modelled using ridge regression method. Along with road geometric and traffic operational data, effective 675 pedestrian perceptions of real-time sense of satisfaction have been used in the model development. A PLOS score model has been developed taking perceived satisfaction score as the dependent variable and factors such as no of lanes, $85^{\text {th }}$ percentile speed of vehicles, volume of through moving vehicles, left turning motorized and non-motorized vehicles, permissible right turning motorized and non-motorized vehicles and pedestrian delay as independent variables. Delays used in PLOS model were estimated by the combination of waiting time delay and vehicle interaction delay. Having $\mathrm{R}^{2}$ value of 0.986 PLOS model was well validated. PLOS scores of six categories were defined by applying Self-Organizing Map (SOM) in Artificial Neural Network (ANN) clustering. Analysis shows that about $75 \%$ of total crosswalks were offering average or above service levels, whereas only $4 \%$ were contributing very poor kind of service to pedestrians in the study areas.
\end{abstract}

Keywords: signalized intersection, pedestrian, delay, level of service.

\section{Introduction}

Mobility of pedestrian as individual or in a platoon along urban road corridors is gradually becoming a major concern to the world community. Often pedestrians are deprived from requiring space for a smooth and safe movement under the influence of complex motorized vehicular movement. Planners and designers are perhaps relent to motorists for which service quality offered to pedestrians is gradually degrading over time. When there is no proper guidelines available to assess service quality of pedestrians, developers use the traditional resource and technology for the road infrastructure development. In developing countries, though pedestrians' population is proportionately high and facilities developed for their use is of course not sufficient. Crossings at signalized intersections are critical locations where pedestrians spent sufficient time of total walk time. These are locations where maximum unwanted

\footnotetext{
${ }^{3}$ Corresponding author: bhuyanp@nitrkl.ac.in
} 
hazards occur in which pedestrians involve predominately. This study has given sufficient effort to define service levels of urban signalized intersection crossings for pedestrian use under the influence of mixed traffic flow. Where mixed traffic conditions are predominant both vehicle and pedestrian share the same space at intersections. Hence leads to pedestrian-vehicular interaction mainly due to the traffic violation behavior of pedestrians. The reason for violation behavior of pedestrians are the arrival pattern of pedestrians, high traffic volume, longer cycle time with no exclusive phase plan for pedestrians in cycle length design. In order to provide a pedestrian friendly facility, it is essential to measure the quality of service in terms of Pedestrian Level of Service (PLOS) provided by the intersection that will make crossing comfortable and safe.

A number of researches have been carried out to asses PLOS in different environments. Some of the background studies are described as follows. Past studies reveal that quantitative model was developed to define six pedestrian service levels ranging from $A$ to $F$, where PLOS A represents the most pedestrian friendly streets in contrast, service level $\mathrm{F}$ represents incomplete streets that fail to provide even the minimum facilities (Sarkar, 1993; Khisty, 1994). Liteture shows that perceived safety for the pedestrian and PLOS decreases with the increase in number of turning vehicles (Muraleetharan et al., 2005). A pedestrian LOS model developed by (Landis et al., 2005) at signalized intersections based on perceived safety and comfort and delay shows that PLOS is mainly affected by vehicle turning right on red. Pulugurtha and Repaka indicated that pedestrian activities can be estimated by considering population, total employment, urban residential area, and the number of transit stops (Pulugurtha and Repaka, 2008).
Highway Capacity Manual (HCM, 2010) and (NCHRP, 2008) methods for analyzing pedestrian LOS are based on the evaluation of measures of effectiveness like pedestrian density, capacity, flow, space, delay, etc.

Conflicts between the right-turning vehicle and the pedestrian coming from the right in the crosswalk in Japan and found that the time lag is constantly re-evaluated during the right turn by the driver, and the driver slows and enters the crosswalk behind the pedestrian, if the time lag at the conflict point is less than 2 seconds (Hagiwara et al., 2008).

Also, the braking location of drivers who braked to avoid conflict with the pedestrian after starting was $10.3 \mathrm{~m}$ before the conflict point. Use existing traffic signal equipment to develop pedestrian performance measures that provide accurate, relevant information with little investment has been developed (Hubbard et al., 2008). Investigated shows the effect of traffic turning right on green for the evaluation of PLOS using binalry logit model (Hubbard et al., 2009). Pedestrian platooning significantly influences the average pedestrian delay compared with random arrivals, when pedestrians are going through several pedestrian signals (Wang and Tian, 2010). Researchers estimated a unique method which may explain a realistic representation of left turning maneuvers to assess the pedestrian safety with the consideration of conflicts with left turning vehicles (Ahhajyaseen $e t$ al., 2012). Pedestrian crossing behavior at signal has been investigated and a microscopic simulation model developed for crossing behavior based on social force theory and desired direction model nearly represent the crossing behaviour (Zeng et al., 2014).

Importance of right-turn capacities at low pedestrian volumes and the effect of turning 
vehicle and bicycle volume on service levels of pedestrian at signalized intersections were examined (Chen et al., 2008; Bian et al., 2009). Observed has been carried out for seven selected intersections in Delhi, India and realized that pedestrian violation occurs due to impatient with increase in signal waiting time (Tiwari et al., 2007). Thus to decrease the probability of pedestrian being hit by motor vehicle, waiting time for pedestrian should be reduce. Studies about pedestrian crossing behavior at signalized intersection with and without countdown displays were carried out; and countdown display was used to reduce pedestrian crossing during red light traffic (Lipovac et al., 2012). In order to estimate pedestrian delay at signalized intersection, by taking the field data of China, a pedestrian delay model was proposed based upon pedestrian signal non-compliance and also the use of Monte Charlo model at intersections was investigated providing various kind of pedestrian facilities (Li et al., 2005; Yang et al., 2005). Under mixed traffic conditions a model for the pedestrian level of service has been developed by considering pedestrian delay and the perception on safety and comfort (Nagraj and Vedagiri, 2013). Analysed about pedestrian safety at intersections also been studied with respect to the safety margin and gap accepted by pedestrian considering different aspects like age, gender, carrying baggage as well as crossing pattern (Jain et al., 2014). Empirical test for the renowned delay models taking a case study on the city center of Bologna, Italy shows that simple models relaying only on cycle timing and geometric parameters are not adequate to represent the actual delay (Mantecchini and Paganelli, 2015). A numerical method has been evaluated for determining crossing delay that applies to crossings of any number of stages and multiple walk intervals per cycle (Furth and Wang, 2015). A traffic dynamic model was developed for an intersection using $\mathrm{M} / \mathrm{M} / 1$ queueing theory and the proposed model was based on simulation of queues, where service was provided with a certain rate (Soh et al., 2007). The task of grouping a set of similar objects in the same group is known as clustering. Self-organizing neural network pattern recognition method was applied to classify highway traffic states into some distinctive cluster centers (Yang and Qiao, 1998). In order to detect and count the vehicles plying on roads from the video graph data Artificial Neural Network (ANN) has been used (Al-Garni and Abdennour, 2008). Methods has been developed by combining ANN and Genetic Algorithm and studies about back propagation Neural Network traffic flow model was carried out (JianMing, 2010; Cetiner et al., 2010).

Although in past studies an extensive work has been done on LOS for pedestrian at signalized intersections, but a limited attention has been paid for heterogeneous traffic flow condition. Therefore, this study aims to develop a suitable delay model for mixed traffic condition and to provide a pedestrian LOS model for developing countries with the consideration of perceived pedestrian data.

\section{Methodology}

The key steps concerned with this study are: selection of factors affecting PLOS at signalized intersections, development of delay model and PLOS score model, classification of PLOS scores into various service categories. Methodology for this study is applied through four steps that determine the pedestrian service measures at signalized intersections. The steps are illustrated in Fig. 1. 


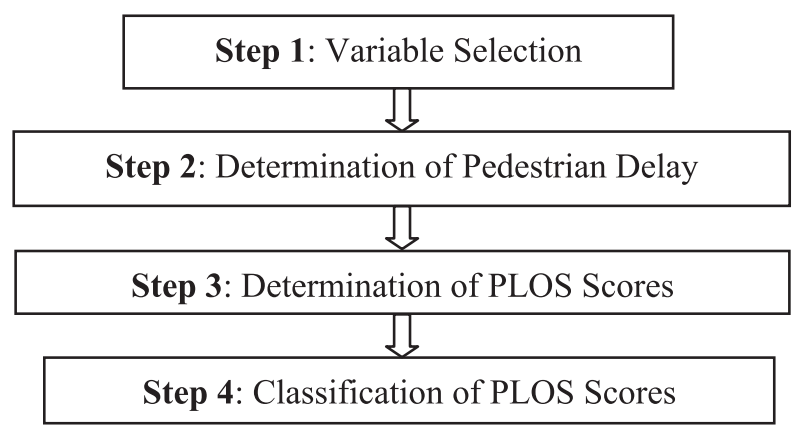

Fig. 1.

Framework of the Study Methodology

\subsection{Selection of Factors Affecting PLOS at Signalized Intersection}

In this study both qualitative and quantitative factors have been selected to develop PLOS model for signalized intersection. As level of satisfaction is a qualitative factor, it can be directly measured by asking the road users about their experience while crossing intersections. For this purpose a questionnaire comprising of 45 questions has been prepared and used for perception survey. In this survey, participants were asked to rate their perceived satisfaction level on a 1-6 scored scale with regard to safety, comfort, traffic influence, maintenance of the road and aesthetics while crossing signalized intersections. Here rating 1 means very much satisfied with the existing facility, similarly, 2- satisfied, 3- somewhat satisfied, 4-somewhat dissatisfied, 5-dissatisfied and 6 - very much dissatisfied. Users' ratings on all questions are averaged and this value is used as the dependent variable in developing PLOS model.

It is noticed from field observations that crossings of pedestrians are affected by both motorized and non-motorized vehicles at signalized intersections. Crossings of pedestrians are not only affected by through traffic, but also left turn and permissible right turn vehicles create conflicts. Following are different quantitative factors which have an effect on PLOS estimation at signalized intersections:

- $\quad$ Number of lanes crossed (1);

- $85^{\text {th }}$ percentile speed of motorized vehicles $(\mathrm{km} / \mathrm{hr})\left(\mathrm{S}_{\mathrm{p}}\right)$;

- $\operatorname{Delay}(\mathrm{D})$;

- Number of left turning motorized vehicles (PCU/15min) $\left(\mathrm{V}_{\mathrm{m}, \mathrm{lt}}\right)$;

- Number of permissible right turning motorized vehicle (PCU/15min) $\left(\mathrm{V}_{\mathrm{m}, \mathrm{rt}, \mathrm{per}}\right)$;

- Number of motorized vehicles through movement (PCU/15min) $\left(\mathrm{V}_{\mathrm{m}, \mathrm{th}}\right)$

- Number of left turning non-motorized vehicle (PCU/15min) $\left(\mathrm{V}_{\mathrm{nm}, \mathrm{lt}}\right)$;

- Number of permissible right turning non-motorized vehicle $\left(\mathrm{V}_{\mathrm{nm}, \mathrm{rt}, \mathrm{per}}\right)$;

- Number of non-motorized vehicle through movement $\left(\mathrm{V}_{\mathrm{nm}, \mathrm{th}}\right)$.

\subsection{Delay Analysis}

Usually the interaction between vehiclepedestrians at signalized intersection leads to delay for both. PLOS model developed in this 
study helps in assessing operational quality of service provided by crosswalks at signalized intersections. Average delay a pedestrian encountered is assumed to be one of the most important parameters in PLOS model development. Hence, accurate estimation of pedestrian delay is a prerequisite.

\section{Delay model}

Pedestrian delay includes mainly two types of delay such as waiting time delay $\left(\mathrm{D}_{\mathrm{PWT}}\right)$ and pedestrian vehicle interaction delay $\left(\mathrm{D}_{\mathrm{PVI}}\right)$ during crossing. As pedestrians while waiting during non-green phase before crossing the road, forms queue at the waiting place, waiting time delay can be computed using queuing concept. In this study waiting time delay has been evaluated using the $M / M / 1$ queuing concept and vehicle interaction delay has to be computed by modelling the factors affecting pedestrian-vehicle interaction. Delay model for signalized intersection is as follows:

$$
D=D_{P W T}+D_{P V I}
$$

Where $\mathrm{D}=$ pedestrian delay at signalized intersection;

$\mathrm{D}_{\mathrm{PWT}}=$ waiting time delay;

$\mathrm{D}_{\mathrm{PVI}}=$ pedestrian vehicle interaction delay. Different delays are described below:

(a) Pedestrian Waiting time delay $\left(D_{P W T}\right)$ :

In general, in a queuing system, customers for the queuing system are generated by an input source. The customers are generated according to a statistical distribution and the distribution describes their inter-arrival times, in other words, the times between arrivals of customers. The basis on which the customers are selected is called the queue discipline. The waiting time delay in this study is modelled based on $M / M / 1$ queuing theory. Poisson distribution usually makes a good fit for the memory less nature of the exponential distribution as the arrival rate of traffic is random in nature.

Vehicles arrive at a single-server facility with mean arrival rate $\lambda$ (vehicles per unit time) and the inter-arrival times between vehicles are independent and identically distributed with mean $1 / \lambda$. Considering traffic arrival and service times at a given intersection as independent random variables, with known distributions, vehicles enter into the system with arrival rate (according to a Poisson process). The average waiting time delay of a pedestrian in the queue at the signalized intersection is:

$D_{P W T}=\frac{\rho}{(\mu-\lambda)}$

Where, $\rho=$ service utilization, $\lambda=$ Arrival rate, $\mu=$ Service rate.

(b) Pedestrian Vehicle interaction delay $\left(D_{P V I}\right)$ :

During pedestrian crossing, delay mainly occurs due to interaction with vehicles due to left turning and permissible right turning of vehicles or other pedestrians. In some cased it is observed that interaction occurs due to the violation nature of drivers and pedestrian during green and non-green phase respectively. Therefore pedestrian vehicle interaction can also be considered as the pedestrian crossing time delay. Pedestrian and vehicle interaction delay is mainly based on parameters like age, platoon size, violating flow rate, and speed of pedestrian. Violating flow rate $\left(\mathrm{V}_{\mathrm{f}}\right)$ can be calculated by observing the number of interacting vehicles as given by the following equation:

Violating flow rate $\left(\mathrm{V}_{\mathrm{f}}\right)=\frac{I V}{t_{v}-t_{p}}$ 
Where IV = Number of interacting vehicles; $\mathrm{t}_{\mathrm{v}}=$ Arrival time of interacting vehicle; $t_{p}=$ Arrival time of pedestrian.

Pedestrian-vehicle interaction influencing parameters are analyzed using ANOVA test to examine the importance and with the help of linear regression analysis a model has been developed for vehicle interaction delay.

\subsection{PLOS Model Development for Signalized Intersections}

In order to check the collinearity among the parameters influencing PLOS variance inflation factor (VIF) has been calculated by using the formula:

$$
V I F=\frac{1}{1-R^{2}}
$$

Here $\mathrm{R}^{2}$ is the parameters of determination of the model. VIF $\geq 10$ shows the multicollinearity of the coefficients and high multicollinearity implies to increase standard errors of estimation and often provides confused and misleading results. This problem can be resolved with the help of ridge regression analysis by minimization of mean square error with the addition of penalty term. The solution of ridge regression can be found by:

$\hat{a}=\left(X^{\prime} X+\lambda I\right)^{-1} X^{\prime} Y$

when $\lambda$ tends to 0 , we get the linear regression estimate;

when $\lambda$ tends to $\infty$, we get $\hat{a}^{\text {ridge }}=0$;

$\lambda \geq 0$ is a turning parameter, which controls the strength of the penalty term.

Ridge regression performed particularly to provide true coefficients for the PLOS model development.

\subsection{Classification of PLOS Scores Using the SOM in ANN Cluster Analysis}

Different PLOS scores model values for each study sites are calculated and classified to get six pedestrian service categories i.e. A-F. Here scores for signalized intersection are classified using Self-Organizing Map (SOM) clustering technique. Among various types of Artificial Neural Network (ANN) algorithms, in this study SOM is used for clustering of PLOS scores because of its inherent capability to learn the pattern of input. IN this classification in the initial step input data are compared with all the input weight vectors $m_{i}(t)$ and the Best Matching Unit $(B M U)$ i.e. the node having the lowest Euclidean distance with respect to the input pattern $x(t) . B M U m_{c}(t)$ is identified by:

For All $i,\left\|x(t)-m_{c}(t)\right\| \leq \| x(t)-m_{i}(t)$

In the next step Weight vectors of BMU are updated as:

$m_{i}(t+1)=m_{i}(t)+\alpha h_{b(x) i}\left(x(t)-m_{i}(t)\right)$

Where $h_{b(x)}$ is the neighborhood function, which is:

$h_{b(x)}=\alpha(t) e^{\left(-\frac{\left\|r_{i}-r_{b(x)}\right\|^{2}}{2 \sigma^{2}(t)}\right)}$

Where,

$0<\alpha(t)<1$ is the learning rate factor which decreases with each iteration;

$r_{i}$ and $r_{b(x)}$ are the locations of neuron in the input lattice;

$\alpha(t)$ defines the width of the neighborhood function;

The steps were repeated iteratively till the pattern in input was processed. 


\section{Study Area and Data Collection}

In order to cover the typical condition of signalized intersections, data were collected from traffic signals having any practical number of pedestrians were using paths to cross. Data were collected from 45 signalized intersections of 8 Indian mid-sized cities (Bhubaneswar, Cuttack, Raipur, Ranchi, Jamshedpur, Tirupati, Vijayawada and Vizianagaram). The population of these cities varies from 0.3 to 1.2 million. Study sites are shown in Fig.2. Selected intersections have varying road geometric with number of approach legs varies from 3 to 5 and distance to cross intersections ranging from $7 \mathrm{~m}$ to $25 \mathrm{~m}$. Some of the cross walks were having well marked zebra crossing where others don't. Selected roads were of major roads, secondary arterial roads and some were of minor roads. Traffic on main carriageway was observed to be highly heterogeneous. Quantitative data regarding traffic flow and road geometric details of major and minor approach roads as well as qualitative data regarding perceived satisfaction level of cross pedestrians were collected. Hourly traffic volume data were collected for major and minor roads of each intersection.

Qualitative data were collected by perception survey using a questionnaire designed for this study. At each intersection around 20 pedestrians were asked about their experience regarding the efficacy of the pedestrian facility. At first participants were convinced about the purpose of the survey and then they were asked to rate satisfaction level on issues related to facility service qualities through several questions. Effectively 675 participants gave their full response till the last part of the questionnaire. These numbers of qualitative data samples were used for analysis purpose. From the total pedestrians participated, $55.25 \%$ are male and $44.75 \%$ are female. Age wise participants were grouped into three categories, i.e. $38.66 \%$ young (15-35 years), $36.59 \%$ middle age ( $35-55$ years) and $24.74 \%$ old (above 55 years).
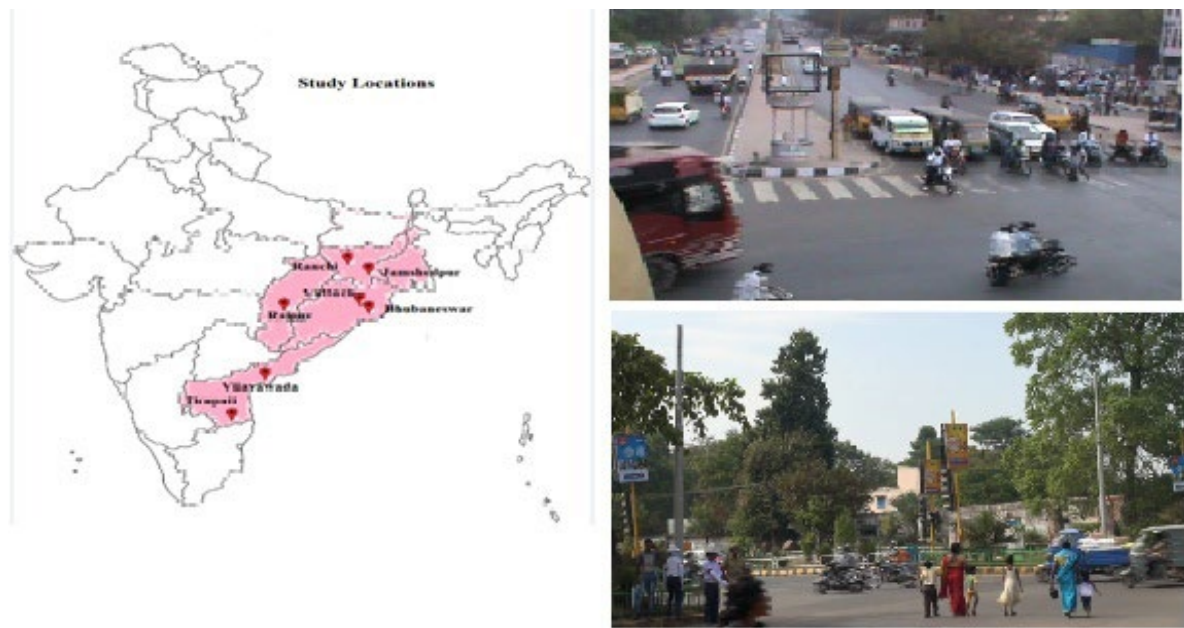

Fig. 2.

Study Locations of Selected Cities of India 
Traffic operational and geometric data of each signalized intersection were collected with video recordings and field observations. Intersection approach roads having number of lanes varying from 2 lanes to 6 lanes are considered in this study. It has been observed that at the study sites hourly volume of motorized vehicles is about 416 PCU/lane to 1752 PCU/lane, where as nonmotorized vehicle volume (by nos.) is 28 to 372. Crossing pedestrian movement is noted as 56 to 316 per hour.

Cycle length at intersections varies from $55 \mathrm{sec}$ to $215 \mathrm{sec}$, red time from $20 \mathrm{sec}$ to 148 $\mathrm{sec}$ and green time from 22 to $65 \mathrm{sec}$. Speed of the vehicles is captured by radar gun and analysis shows that $85 \%$ speed of vehicles varied from $25.25 \mathrm{~km} / \mathrm{h}$ to $40 \mathrm{~km} / \mathrm{h}$. The lower speed range is due to a significant percentage of slow moving vehicles under the mixed traffic flow during peak hours. Numbers of left turns, permissible right turn and through moving vehicles are also observed separately for the detail analysis purpose.

\section{Results and Discussion}

As delay is one of the most important variable in PLOS modelling at signalized intersections, in this part of the study, evaluation of the proposed delay model and its comparison with other delay models has been described. Along with delay relationship between other quantitative variables with overall perceived satisfaction has been analyzed. After analyzing the importance of variables, PLOS score model has been developed for signalized intersection using ridge regression technique. Output scores of PLOS model are then classified into six categories (A-F) applying SOM in ANN clustering technique.

\subsection{Pedestrian Delay Model Development}

It has been observed that pedestrian-vehicle interaction delay mainly occurs in noncompliance of traffic rules by pedestrians or vehicles. Mostly pedestrian violation nature reduces the total delay, in fact, some minute delay should be there yet. To measure the pedestrian-vehicle interaction delay time some assumptions are made. Accordingly pedestrian group size, violating flow rate, age, gender, walking speed and number of pedestrians has considered in analyzing the pedestrianvehicle interaction. But only platoon size (PS), violating flow rate $\left(\mathrm{V}_{\mathrm{f}}\right)$ and walking speed $\left(\mathrm{S}_{\mathrm{p}}\right)$ has shown the significance (P-value) of $0.000,0.000$ and 0.002 respectively as output with the observed pedestrian- vehicle interaction time in the field. From the analysis, pedestrian-vehicle interaction time delay has been calculated using the following formula developed in this study:

$\mathrm{D}_{\mathrm{PVI}}=0.68(\mathrm{PS})+1.308\left(\mathrm{~V}_{\mathrm{f}}\right)+4.347\left(\mathrm{~S}_{\mathrm{p}}\right)-0.935$

The total delay has been calculated by the summation of waiting time delay, crossing time delay and vehicle interaction delay using eq. (1).

Comparison of Proposed Delay Model:

Field delay of different intersections are calculated by extracting the observation data from the field as well as from the video. The parameters considered are arrival time, crossing time of a pedestrian at intersection, total number of pedestrians and field crossing time that each individual pedestrian takes to cross the road. During the red phase of pedestrian the arrival time of each pedestrian per minute at the cross walk location has noted with the help of stop watch. Similarly, the time taken to cross the 
road also noted as same and at field crossing time of a pedestrian has measured during green phase. The field delay for a pedestrian at signalized intersection is as follows:

Observed Field Delay $=\frac{(\text { Crossing time }- \text { Arrival time }) \text {-Field crossing time }}{\text { Total number of pedestrians }}$

After getting the field delay, the delay values using different existing methods by applying observed field datas also calculated. Then the proposed pedestrian delay model (PDM) in this study compared with other existing models like HCM delay model (HCMD) (6), (Braun and Roddin, 1978) delay model (BRD), Marisamynathan and Vedagiri delay model (MVD), Deterministic queuing model (DD), Steady state stochastic delay model (SD) as shown in Fig. 3.

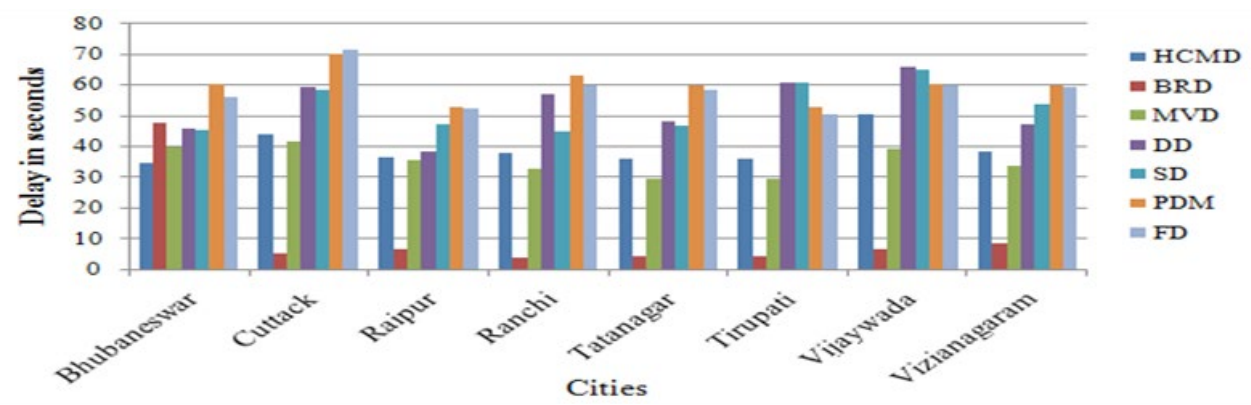

Fig. 3.

Comparison of Existing Models with Field Delay and Pedestrian Delay Model

It has been observed that the proposed pedestrian delay model (PDM) values are found to be nearer with observed field delay (FD) rather than other existing models under heterogeneous traffic flow.

\subsection{Effects of Quantitative Parameters on Perceived Satisfaction Scores}

Microscopic analysis was carried out to study the interrelationship between users perceived satisfaction scores (highest satisfaction score means least satisfied with an item) and quantitative measured parameters contributing to signalized intersection service levels. Typical scatter plots of these parameters are shown in Fig. 4. Study examination reveals that satisfaction score linearly increases with the increase in vehicular speed. As the higher range of scores represents dissatisfaction of users, it means to say that walkers feel unsafe and discomfort when vehicles move at higher speeds. It is observed that when the volume of vehicles (both motorized and non-motorized) increase on the main carriageway conflicts between vehicles and pedestrians crossing at intersections also increase which causes the dissatisfaction to pedestrians. Sometime due to high traffic volume pedestrian delay at intersections became more and pedestrian have to wait for a long time to cross. A logarithmic incriminate with $\mathrm{R}^{2}$ value of 0.708 has been observed between pedestrian delay and perceived satisfaction scores. 


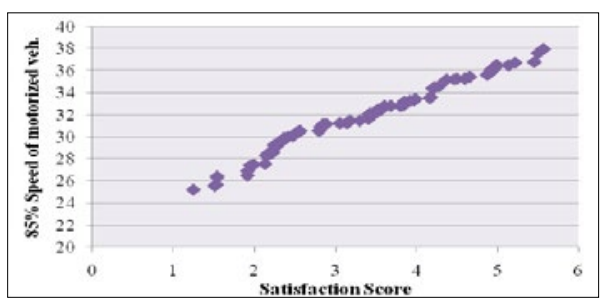

(a)

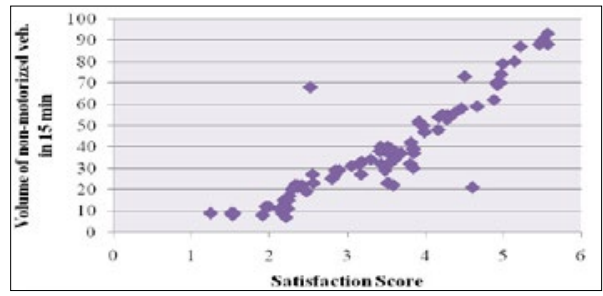

(c)

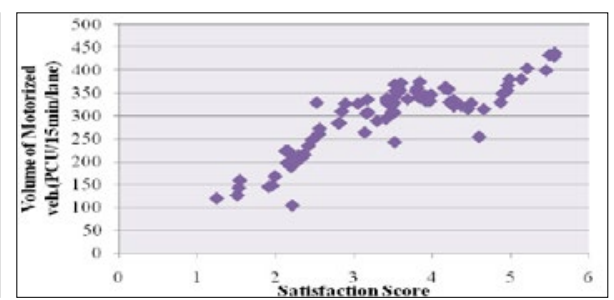

(b)

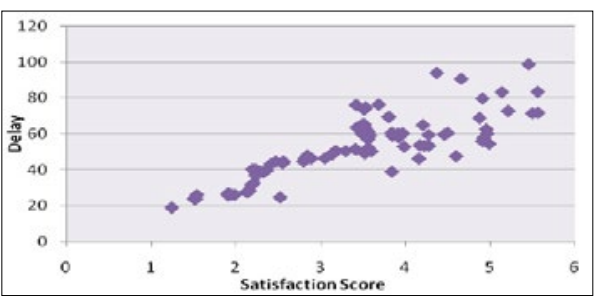

(d)

Fig. 4.

Correlation of Perceived Satisfaction Score with (a) 85\% Speed of Motorized Vehicles, (b) Volume of Motorized Vehicles, (c) Volume of Non-Motorized Vehicles, (d) Pedestrian Delay

\subsection{PLOS Model Development}

After examining the correlation between quantitative parameters and perception based satisfaction score, analysis has been carried out to establish a PLOS model for signalized intersection by taking user perception score as dependent variable.

Analysis shows that all the quantitative variables taken such as no of lanes, $85^{\text {th }}$ percentile speed of vehicles, left turning vehicle, permissible right turning vehicle, the volume of through vehicles and pedestrian delay are significant for model fitting as $P$-value for each parameter are $<0.05$. The proposed model for satisfaction levels of pedestrians configured in the following format:
PLOS scores for signalized intersection $=$ $c+a_{1} l+a_{2} S_{p}+a_{3}\left(V_{m, l t}+V_{m, r t, p e r}\right)+a_{4} V_{m, t h}+$ $+a_{5} V_{n m, t h}+a_{6} \ln V_{n m, l t}+a_{7} e^{0.428 V_{n m, r, p e r m}}+a_{8} \ln D$

It has been observed that during linear regression analysis the VIF for PLOS variables are greater than 10 indicating serious multicollinearity. Variables are analyzed via ridge regression technique with the help of Statgraphics software. Adjusting $\lambda$ (ridge parameter $)=0.15$, estimated coefficients and VIF values are given in Table 1 . From the ridge regression analysis $\mathrm{R}^{2}$ for this model is found to be 0.931 with a mean absolute error of 0.1423 that explains about the significance of the model. Using $40 \%$ of total datasets proposed PLOS score model has been validated successfully with significance of $\mathrm{R}^{2}=0.986$. 
Table 1

PLOS Score Model Coefficients and Variance Inflation Factor (VIF)

\begin{tabular}{|c|l|l|l|}
\hline $\begin{array}{l}\text { Model } \\
\text { Parameters }\end{array}$ & Coefficients & VIF & P value \\
\hline $\mathrm{c}$ & -3.89 & - & 0.000 \\
\hline $\mathrm{a}_{1}$ & 0.324 & 1.07765 & 0.021 \\
\hline $\mathrm{a}_{2}$ & 0.108 & 0.79595 & 0.000 \\
\hline $\mathrm{a}_{3}$ & 0.008 & 0.91737 & 0.03 \\
\hline $\mathrm{a}_{4}$ & 0.0013 & 1.29136 & 0.006 \\
\hline $\mathrm{a}_{5}$ & 0.006 & 1.16603 & 0.016 \\
\hline $\mathrm{a}_{6}$ & 0.205 & 1.28194 & 0.0089 \\
\hline $\mathrm{a}_{7}$ & 0.0012 & 1.23161 & 0.000 \\
\hline $\mathrm{a}_{8}$ & 0.442 & 1.34867 & 0.019 \\
\hline
\end{tabular}

\subsection{Classification of Plos Scores}

The PLOS model output gives the predicted pedestrian satisfaction scores at signalized intersections. In order to define ranges of PLOS scores of six categories (A-F), SOM in ANN clustering technique has been applied in this study. Ranges of PLOS categories obtained from this classification are shown in different colors and legends in the Fig. 5.

From the above figure it has been observed that PLOS ' $\mathrm{A}$ ' is having score of less than ' 2 ' which describes about the best service quality. In PLOS 'A' condition pedestrians have the ability to move in the desired path without alternating their movements.
PLOS 'B' ranges from score value ' 2 to 3 ' where occasional walkers need to avoid their paths to avoid conflicts. In case of PLOS ' $C$ ' ranging from ' 3 to 3.6 ' frequently requirement of adjustment in a path can be observed; whereas in PLOS ' $D$ ' ranging from ' 3.6 to 4.11 ', passing of slower pedestrian is somehow difficult. Service score of ' 4.11 to 5 ' belongs to PLOS 'E' where speed of pedestrian is restricted and pedestrian waiting time is more. PLOS ' $F$ ' represents the worst state of service to walkers having PLOS score greater than ' 5 '. In PLOS F condition pedestrian has to wait for a long to cross the intersection. In this case pedestrian speed is severely restricted and frequent contact with other road users has been observed.

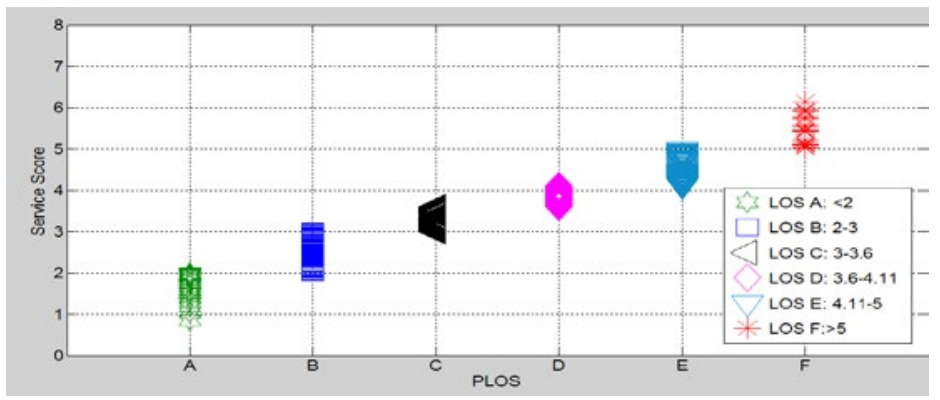

Fig. 5.

PLOS Categories of Signalized Intersection 


\subsection{Comparison of Proposed Model with Existing Models}

PLOS score ranges from the current study categorized using the SOM in ANN clustering technique has been compared to the already defined ranges of other existing models as shown in Table 2. Here in this study the lower and upper perceived value varies between 1 and 6 . In some cases signalized crosswalks offer excellent service quality of ' $A$ ' and its service value is found to be 2 or below it. Predicted service levels on 10 crosswalks of signalized intersections based on a certain set of geometric and traffic conditions were compared with the estimated service levels of same crosswalks using NCHRP and Yang Bian model. Service levels using all these three models on selected 10 crosswalks were compared with the average perceived PLOS as shown in the Table 3. In order to compare threshold values of model output and existing model with actual field satisfaction (perceived satisfaction) PLOS categories of taking data sets of random 10 sites, estimation has been carried out by applying two existing PLOS models (NCHRP, Yang Bian) and proposed model. PLOS using different models for signalized intersections are as shown in Table 3.

Table 2

Comparison of Ranges of PLOS Categories (A-F) for Signalized Intersection

\begin{tabular}{|c|c|c|c|}
\hline PLOS & NCHRP & Yang Bian & $\begin{array}{c}\text { Proposed } \\
\text { Model }\end{array}$ \\
\hline A & $\leq 2$ & $\leq 1.5$ & $\leq 2$ \\
\hline B & $>2-2.75$ & $>1.5-2.5$ & $>2-3$ \\
\hline C & $>2.75-3.5$ & $>2.5-3.5$ & $>3-3.6$ \\
\hline D & $>3.5-4.25$ & $>3.5-4.5$ & $>3.6-4.11$ \\
\hline E & $>4.25-5$ & $>4.5-5.5$ & $>4.11-5$ \\
\hline F & $>5$ & $>5.5$ & $>5$ \\
\hline
\end{tabular}

Table 3

Comparisons of Perceived PLOS with Predicted Service Levels of Existing and Proposed Models

\begin{tabular}{|c|c|c|c|c|}
\hline Sites & $\begin{array}{c}\text { Perceived Service } \\
\text { Level }\end{array}$ & NCHRP & Yang Bian & Proposed Model \\
\hline 1 & C & B & F & C \\
\hline 2 & A & B & E & A \\
\hline 3 & C & C & F & C \\
\hline 4 & A & B & D & F \\
\hline 5 & F & E & F & E \\
\hline 6 & F & E & D & E \\
\hline 7 & A & D & F & B \\
\hline 8 & B & C & D & A \\
\hline 9 & A & B & F & $20 \%$ \\
\hline
\end{tabular}


Data analysis of perception survey and subsequently PLOS model development using several variables revealed that predicted PLOS scores are not only affected by the motorized vehicles but also by the presence of non-motorized vehicles. In NCHRP model, although many important factors have been considered but the influence of non-motorized traffic is not cleared. In the proposed model due attention has been paid for both motorized and non-motorized vehicles moving in different directions (left turn, right turn and through) along with no of lanes, speed of vehicles and pedestrian delay in heterogeneous traffic flow condition. Comparison of model outputs shows that PLOS categories estimated by applying the proposed model give more compatible results with field perceived satisfaction level than existing model outputs. Predicted outputs using NCHRP and Yang Bian models show that only $10 \%$ and $20 \%$ matching with field observed service levels on 10 selected sites. Hence the proposed model is more suitable to estimate the pedestrian satisfaction levels at signalized intersections in mixed traffic condition.

\section{Conclusion}

Study findings revealed that pedestrian satisfaction at signalized intersection is a complex term which depends on several parameters from which delay on crosswalks is an important one. Analysis on selected 45 crosswalks shows that total pedestrian delay increases linearly with increase in waiting time delay with a significance value of $\mathrm{R}^{2}=0.986$. When pedestrian speed increases up to 1.3 $\mathrm{m} / \mathrm{sec}$ to avoid conflict, then actual crossing time becomes less than the ideal crossing time and hence total delay decreases. Total delay is $56.38 \mathrm{sec}$ for $7.87 \mathrm{sec}$ interaction time delay and $8.3 \mathrm{sec}$ interaction time delay has given, total delay of $55.62 \mathrm{sec}$. So with the increase in pedestrian-vehicle interaction time, total delay decreases logarithmically. The average value of Pedestrian-vehicle interaction delay occurs during non-compliance of traffic rules by the pedestrian or vehicle has been observed as $78 \mathrm{sec}$.

A PLOS score model developed using several variables was validated with significance of $\mathrm{R}^{2}=0.986$. Different ranges of PLOS scores show that intersections having score value less than 2 provide best quality of service with a pedestrian delay of $<20$ seconds where as score of greater than 5 with delay of $>70$ seconds are having the worst facility to pedestrian. Increase in volume of left turning and permissible right turning leads to conflict at the intersection due to which satisfaction level decreases. At some intersections sufficient pedestrian green time up to 70 seconds has been provided so that crossing people will get better service. It is also noted that when the volume of left turn and permissible right turn vehicle volume is less than $120 \mathrm{pcu} / \mathrm{h}$ and $36 \mathrm{pcu} / \mathrm{h}$ pedestrian feel comfortable to cross. These may be the reason, that $20 \%$ and $24 \%$ of total study intersections were having PLOS 'A' and ' $\mathrm{B}$ ' respectively. Above $70 \%$ of participants suggested that in cycle length design exclusive phase plans for pedestrians should be provided.

Maximum of $30 \%$ sites are having moderate service quality, i.e. of PLOS ' $\mathrm{C}$ ' category as although pedestrians were having difficulties due to conflict with vehicles but they were able to cross the intersection by increasing their speed. In some cases, pedestrian delay was more, and if walker wants to cross quickly by violating traffic rules they have to face conflict with vehicles. Results show $6 \%$ and $4 \%$ of the total study area were having PLOS 'E' and ' $F$ ' of poor service quality for pedestrian during crossing. Comparison of 
existing and proposed PLOS model values with perceived satisfaction score shows that proposed model is matching $90 \%$ with the field value. From the study it has been noted that in present condition from the total study areas more than $75 \%$ of the signalized intersections are providing average or better of its service levels to the pedestrians. Predicted values using the delay model developed in this study show that the delay will increase up to $30 \%$ with a $10 \%$ increase in pedestrian service rate. In that condition more than $50 \%$ of the intersection will provide below average service. Therefore facilities need to be improved to provide a pedestrian friendly environment with better service levels. The proposed PLOS model can be used by the roadway planners and designers to measure the performance of existing signalized intersection in heterogeneous traffic flow condition for mid-sized cities. Regarding large cities having more than million populations are to be investigated in further studies.

\section{References}

Ahhajyaseen, W.K.; Asano, M.; Nakamura, H. 2012. Estimation of left-turning vehicle maneuvers for the assessment of pedestrian safety at intersections, IATSS Research 36(1): 66-74. DOI:10.1016/j.iatssr.2012.03.002.

Al-Garni, S.; Abdennour, A. 2008. A neural network based traffic flow evaluation system for highways, Journal of King Saud University of Engineering Sciences 20(1): 37-46.

Bian, Y.; Ma. J.; Rong. J; Wang, W.; Lu, J. 2009. Pedestrians' level of service at signalized intersections in China, Transportation Research Record: Journal of the Transportation Research Board 2114: 83-89. DOI: 10.3141/2114-10.

Braun, R.R.; Roddin, M.F. 1978. Quantifying the benefits of separating pedestrians and vehicles. NCHRP Report 189. Transportation Research Board. Washington, D.C. 127 p.
Cetiner, B.G.; Sari, M.; Borat, O. 2010. A neural network based traffic-flow model, Mathematical and Computational Applications15(2): 269-278. DOI:10.3390/mca15020269.

Chen, X.M.; Shao, C.F.; Hao, Y. 2008. Influence of Pedestrian Traffic on Capacity of Right-Turning Movements at Signalized Intersections, Transportation Research Record: Journal of the Transportation Research Board 2073: 114-124. DOI: 10.3141/2073-13

Furth, P.G.; Wang, Y.D. 2015. Delay Estimation and Signal Timing Design Techniques for Multi-Stage Pedestrian Crossings and Two-Stage Bicycle Left Turns. In Proceedings of the Transportation Research Board 94th Annual Meeting (No. 15-5365). 1-16.

Hagiwara, T.; Hamaoka, H.; Yaegashi, T.; Miki, K.; Ohshima, I.; Naito, M. 2008. Estimation of time lag between right-turning vehicles and pedestrians approaching from the right side, Transportation Research Record: Journal of the Transportation Research Board 2069: 65-76. DOI: 10.3141/2069-09.

HCM. 2010. Highway Capacity Manual. Transportation Research Board, Washington, D.C.

Hubbard, S.M.; Bullock, D.M.; Mannering, F.L. 2009. Right turn on green and pedestrian level of servive: statistical assessment, Journal of Transportation Engineering 135(4): 153-159.

Hubbard, S.; Bullock, D.; Day, C. 2008. Integration of real-time pedestrian performance measures into existing infrastructure of traffic signal system, Transportation Research Record: Journal of the Transportation Research Board 2080: 37-47. DOI: 10.3141/2080-05.

Jain, A.; Gupta, A.; Rastogi, R. 2014. Pedestrian Crossing Behaviour Analysis at Intersections, International Journal for Traffic and Transport Engineering 4(1): 103-116. DOI: http://dx.doi.org/10.7708/ijtte.2014.4(1).08.

Jian-Ming, C. 2010. Traffic Prediction Based on Improved Neural Network, Journal of Convergence Information Technology 5(9): 85-89. 
Khisty, C.J. 1994. Evaluation of pedestrian facilities: Beyond the level-of-service concept. Transportation Research Record, Transportation Research Board, Washington, D.C. $1438: 45-50$.

Landis, B.; Petritsch, T.; McLeod, P.; Huang, H.; Guttenplan, M. 2005. Video Simulation of Pedestrian Crossings at Signalized Intersections, Transportation Research Record: Journal of the Transportation Research Board 1920: 49-55. DOI: 10.3141/1920-06.

Li, Q.; Wang, Z.; Yang, J; Wang, J. 2005. Pedestrian delay estimation at signalized intersections in developing cities, Transportation Research Part A: Policy and Practice 39(1): 61-73. DOI:10.1016/j.matcom.2005.01.017.

Lipovac, K.; Vujanic, M; Maric, B; Nesic, M. 2012. Pedestrian behavior at signalized pedestrian crossings, Journal of transportation engineering 139(2): 165-172. DOI: 10.1061/(ASCE)TE.1943-5436.0000491.

Mantecchini, L.; Paganelli, F. 2015. Emperical Analysis of Pedestrian Delay Models at Urban Intersections, Contemporary Engineering Science 8(21): 981-990. http:// dx.doi.org/10.12988/ces.2015.57202.

Marisamynathan, S.; Vedagiri, P. 2013. Modeling pedestrian delay at signalized intersection crosswalks under mixed traffic condition, Procedia-Social and Behavioral Sciences 104: 708-717. doi: 10.1016/j. sbspro.2013.11.165.

Muraleetharan, T.; Adachi, T.; Hagiwara, T.; Kagaya, S. 2005. Method to Determine Pedestrian Level-ofService for Crosswalk at Urban Intersections, Journal of the Eastern Asia Society for Transportation Studies 6: 127-136. DOI:10.4028/www.scientific.net/AMM.253255.1936 .

Nagraj, R.; Vedagiri, P. 2013. Modeling Pedestrian Delay and Level of Service at Signalized Intersection Crosswalks under Mixed Traffic Conditions, Transportation Research Record: Journal of the Transportation Research Board 2394: 70-76. DOI: 10.3141/2394-09.
NCHRP. 2008. NCHRP Report 616: Multimodal Level of Service Analysis for Urban Streets. Transportation Research Board, National Research Council, Washington, D.C. 60 p.

Pulugurtha, S.; Repaka, S. 2008. Assessment of Models to Measure Pedestrian Activity at Signalized Intersections, Transportation Transportation Research Record: Journal of the Transportation Research Board 2073: 39-48. DOI: 10.3141/2073-05.

Sarkar, S. 1993. Determination of service levels for pedestrians, with European examples, Transportation Research Record 1405: 35-42.

Soh, A.; Marhaban, M.; Khalid, M.; Yusof, R. 2007. Modelling and Optimization of a Traffic Intersection Based on Queue Theory and Markov Decision Control Methods. In Proceedings of the First Asia International Conference on Modelling \& Simulation (AMS'07). 478-483. DOI: 10.1109/AMS.2007.64

Tiwari, G.; Bangdiwala, S.; Saraswat, A.; Gaurav, S. 2007. Survival analysis: pedestrian risk exposure at signalized intersections, Transportation research part F: traffic psychology and behaviour, 10(2): 77-89.

Wang, X.; Tian, Z. 2010. Pedestrian Delay at Signalized Intersections with a Two-Stage Crossing Design, Transportation Research Record: Journal of the Transportation Research Board 2173: 133-138. DOI: 10.3141/2173-16.

Yang, H.; Qiao, F. 1998. Neural network approach to classification of traffic flow states, Journal of Transportation Engineering 124(6): 521-525.

Yang, J.; Li, Q.; Wang, Z.; Wang, J. 2005. Estimating pedestrian delays at signalized intersections in developing cities by Monte Carlo method, Mathematics and Computers in Simulation 68(4): 329-337.

Zeng, W.; Nakamura, H.; Chen, P. 2014. A Modified Social Force Model for Pedestrian Behavior Simulation at Signalized Crosswalks, Procedia - Social and Behavioral Sciences 138: 521-530. DOI: 10.1016/j.sbspro.2014.07.233. 